anæsthesia. This method is the continuation, looally, of anæsthesia which during an operation has been general.

I claim that it is possible by the infiltration of the area of an operation wound before making that wound, with a solution of quinine and urea bydrochloride, to produce a total absence of any reactionary pain in the area of operation after the patient has recovered from the effects of the general anpesthetic. The method of use is quite simple and within the reach of any operator. The nerve supply of an area being known, it is quite easy to distribute from five to ten c.c. of a 1 per cent. solution over that area, and then proceed with the operation as under ordinary conditions. The patient is first put under a general anæsthetic, and the surgeon proceeds to make multiple injections of a sterilised solution of quinine and urea hydrochloride all over and around the site of his proposed incisions and manipulations.

The effect of these injections is to produce an almost total loss of sensation in the parts for a length of time varying from 24 hours to 6 days, with absolutely no return or vestige of post-operative pain, until healing is complete, and a minimum of operative and post-operative shock. The vitality of the tissues injected is not jeopardised; indeed, the absence of pain abolishes to a large extent reactionary stasis in the tissues, and tends to promote rapid healing. My experience so far has been that from the moment of the completion of an operation until the skin has healed the patient is not conscious of suffering from injury to the parts operated on. If further observations confirm these results the method will confer a boon on delicate and nervous women and others. Crushed limbs and fractures could be made painless and fatal shock averted by the immediate infiltration of the damaged part above the seat of injury with this solution, which is prepared for me by Messrs. Parke, Davis, and Co.

Infiltration anæsthesia by cocaine and suprarenal extract is very often accompanied by the most intense agony when the circulation of the infiltrated part begins to return and sensation is being re-established. This is not the case with quinine and urea hydrochloride anæsthesia. There is no local ischæmia. This method, I suggest, should prove most useful in diseases of the rectum and associated parts, and in compound fractures and crushed limbs, and indeed to obviate pain after injury or operation in any part of the human body accessible to a hypodermic syringe.

I am, Sir, yours faithfully,

F. W. Forbes-Ross, M.D. Edin, E.R.C.S. Eng.

New Carendish-street, W., June 8th, 1912.

\section{BROMIDE OF STRONTIUM IN EPILEPSY.}

\section{To the Editor of THE LANOET.}

SiR, - Some twenty years ago I read in your correspondence columns a letter from a practitioner recommending bromide of strontium in epilepsy. For a week or two afterwards the usual letters appeared from other medical men who had already tried it and found it, in their hands, no better than, or perhaps not so good as the other bromides. I tried it in a few cases in the usual small doses, and in the main I found little difference in the results obtained as compared with the bromides of potassium, sodium, or ammonium. I then experimented in several cases by increasing the dose up to $3 i$, or even more, three times daily, and I have had a large measure of success in very many cases.

As my work for the last 18 or 20 years has consisted of mainly contract practice, I have had exceptional opportunities of giving any particular drug over a lengthened period and observing its effects. I find that the bromide of strontium given in large doses does more effectually sontrol the attacks of epilepsy than the usual bromides, and is more often followed by a cure. To effect this result the treatment must be persisted in over an extended time; in many cases 10 to 12 months' treatment is necessary, and in a few cases even a few months longer. I have found no difficulty in giving it over this extended time, the patients getting into a habit of never missing a dose. Excluding those cases which are of old standing and in which the will power and brain seem impaired, and excluding those due to rellex causes, I find all other cases susceptible to treatment by the strontium bromide. Those which most readily yield a good result are the young adults who have not for an extended period suffered from attacks, but even in middle-aged patients success is usually attained by persistent administration of the drug.

Attacks of epilepsy during the time the patient is under treatment are rare. I have given it in many cases where from its first inception no attacks have occurred, and the patient has gone right on to recovery. In those cases a much shorter period is required for treatment, but I always endeavour, even in the absence of attacks, to keep the patient on the drug as long as I can in reason. I find that though these large doses are well borne by the stomach they cause a great deal of mental depression, but the patient, encouraged by the prospect of recovery and suffering from the recollection of the attacks, readily bears the inconvenience. Acne is much rarer than after the administration of the other bromides. I have given equally large doses of tie three bromides over an extended time, but the results were not so satisfactory or so permanent.

I have now a number of patients who were formerly severe sufferers from attacks of epilepsy, but who for some years have been free from them, and so I presume I may consider them as cured. I think I have waited a reasonable time before rushing into print to lay the results of my experience before my professional brethren. I have no doubt they will experience the same good results when they experiment with the drug in suitably large doses.

I am, Sir, yours faithfull

Lowestoft, June 10th, 1912

M. R. CALLENDER,

\section{THE ESTIMATION OF NICOTINE IN TOBACCO.}

To the Editor of THE LANCET.

SIR, - May we be allowed to correct an error, due to a misunderstanding, in your comments on our recent paper on the Estimation of Nicotine in Tobacco? You state that the solubility of the iodine compound of nicotine affects our method as well as that employed in your laboratory, but this is not so. The compound is insoluble in presence of excess of iodine, and is therefore completely removed in our method; it is only when it is washed with water that its solubility introduces an error. As to the advantages or otherwise of distillation, its tediousness must be admitted; but the suggestion that the minute quantity of potash that may be formed causes loss of alkaloid can hardly be taken seriously, especially when you refer to the results of an experiment which gave an error of 11 per cent. as " accurate."

We are, Sir, yours faithfully,

London, W.C., June 11tb, 191 ?

E. F. HARRISON.

** An error of 11 per cent. of the total nicotine present means, of course, that in a tobacco containing actually 1.00 per cent. of nicotine the method of analysis would give $1 \cdot 10$ per cent. - ED. L.

\section{MALIGNANT DISEASE OF THE UTERUS.}

To the Editor of THW LANCET.

SIR,-In your issue of May 25th Dr. T. W. Eden, writing on cancer of the corpus uteri, makes some interesting statements, but he does not draw from them certain obvious conclusions.

He first says with regard to fecundity in the two kinds of nterine cancer : "The average number of children borne was, in cancer of the cervix $4 \cdot 1$; in cancer of the body $1 \cdot 1$." He then goes on to state that cancer of the cervix is relatively commoner in the poor than the rich, more so than cancer of the body, which he thinks is not. He found that in cases of cervical cancer the proportion of hospital to private patients was 10 to 1 , and in cancer of the body 3 to 1 . He quotes similar opinions of Sir William Sinclair and others, "which," he says, "I share myself, that cancer of the cervix is a disease which falls with especial severity upon the poorer classes, and it is therefore probable that poverty and insanitary conditions of life are predisposing causes of its occurrence. My impression is, although I admit that I am unable to prove it, that this is not the case with cancer of the body.

While doubtless the statements of fact are correct, it is difficult to understand how poverty and insanitary conditions can cause cancer. The explanation, of course, really lies in 